\title{
Incidencia de la jornada escolar en los procesos de socialización infantil
}

\section{School day incidence on children's socialization processes}

\author{
M. Carmen Morán de Castro ${ }^{1}$
}

\begin{abstract}
RESUMEN
El artículo, derivado de la tesis doctoral realizada por la autora, sitúa el punto de análisis en la percepción del tiempo social en general, y particularmente el escolar, como una realidad materializada en significados diversos para las niñas y niños, generadores de tensiones y conflictos en los procesos de socialización infantil. La vida cotidiana de la infancia en sus contextos familiar, extrafamiliar y escolar, está fuertemente mediatizada por las demandas y presiones del tiempo escolar que preside y organiza la vida de los pequeños y sus familias. El artículo analiza esta incidencia tomando como variable fundamental la modalidad de jornada lectiva, unidad temporal que ha usurpado el debate sobre la globalidad de los tiempos escolares y educativos que es, no sólo deseable, sino urgente en el contexto español. En el estudio se aportan los resultados obtenidos en la investigación realizada con alumnado gallego de Educación Primaria, alrededor de las siguientes cuestiones: planificación, administración y gestión del tiempo; percepción de su transcurrir y valoración de su disponibilidad y uso en los ámbitos familiar, escolar y extraescolar. La variable modalidad de jornada escolar (sesión única matinal/sesión partida mañana y tarde) se ha tomado como principal referencia en el análisis.
\end{abstract}

Palabras clave: infancia; socialización; tiempo socia; tiempo escolar; jornada lectiva.

\begin{abstract}
The paper, derived of the Dissertation realised by the author, situates the point of analysis in the perception of social time in general, and particularly
\end{abstract}

1 Doctora en Pedagogía. Profesora de la Facultad de Ciencias de la Educación-Universidad de Santiago de Compostela, España. E-mail: carmen.moran@usc.es. 
in school time, like a reality materialised in diverse meanings for girls and boys, generators of tensions and conflicts in the processes of chilhood socialization. Daily life of childhood in his familiar, extrafamiliar and school contexts, is strongly influenced by the demands and pressures of the time at school that prevailes and organises the life of children and their families. The paper analyzes this incidence taking the modality of the school day as a fundamental variable, temporary unit that has usurped the debate on the entirety of the school time and the educational time that it is, not only desirable, but urgent in the Spanish context. In the study, we show the results obtained in the investigation realised with Galician students of Primary Education, around the following questions: planning, administration and management of time; perception of how time goes and the assessment of his availability and use in the family, school and extraescolar fields. The modality of the school day (only morning session /session split morning and afternoon) variable, has taken the main reference in the analysis.

Keywords: childhood; socialization; social time; school time; school day.

\section{Introducción ${ }^{2}$}

El elemento nuclear del trabajo que se presenta es el estudio del tiempo, referente implacable en la vida de las personas, eje vertebrador del transcurrir diario, donador o limitador de oportunidades materiales y afectivas y, consecuentemente, considerado como el elemento sustancial o núcleo gordiano de muchos de los conflictos en la sociedad apresurada diluída en tiempos líquidos y volátiles (BAUMAN, 2007; MARRAMAO, 2008). Conflictos que son vivenciados no ya en edades maduras, donde los compromisos reales o creados desbordan las posibilidades horarias, sino en la infancia, sumergida en la vorágine adulta de la

2 La tesis doctoral de la que surge este trabajo se inserta en la línea "tiempos sociales, educativos y escolares" del grupo de investigación en Pedagogía Social y Educación Ambiental (SEPA) de la Universidad de Santiago de Compostela, dirigido por el prof. Dr. José A. Caride Gómez.

El grupo SEPA forma parte de la Red de Grupos de Investigación RINEF-CISOC (Red de Investigación en Educación y Formación para la Ciudadanía y la Sociedad del Conocimiento), financiada por el Fondo Europeo de Desarrollo Regional (FEDER), a través de la Secretaría Xeral de Universidades de la Consellería de Educación e Ordenación Universitaria de la Xunta de Galicia, en el marco de la Convocatoria de Ayudas para la consolidación y la estruturación de Unidades de Investigación Competitivas del Sistema Universitario de Galicia, correspondiente al año 2011 (DOG nº 258, do 19 de agosto de 2011). 
conquista del tiempo, aunque no sea precisamente -como desearía el Principito de S. Exupèry- para disfrutarlo caminando lentamente hacia una fuente.

El tiempo es protagonista tenso y problematizador de la vida cotidiana de la infancia, tanto en los ámbitos familiar y extra-familiar, como en el escolar. En los contextos de vida cotidiana como consecuencia de las discrepancias entre los tiempos laborales, escolares y familiares. Es el conflicto de la conciliación horaria (CARDÚS, 2003), símbolo de la era que vivimos, que afecta de forma negativa a nivel individual, familiar y comunitario. Sus consecuencias se visibilizan en la apreciación de altos grados de ansiedad, estrés, insatisfacción; en las dificultades de disfrute de tiempo propio y libre, de expresión de afectos, de mejora de las condiciones formativas; en el deterioro de la calidad y cantidad de oportunidades para el encuentro y convivencia; en la merma de la cohesión familiar, incremento de incomunicación y aislamiento; en el deterioro de la interacción con iguales, en la restricción de las posibilidades de implicación, participación y ejercicio de la ciudadanía, etc. Un buen número de circunstancias, factores y variables altamente incidentes en la generación de desigualdades de oportunidades y consiguiente vulnerabilidad social.

En el contexto escolar español se ha incrementado la perspectiva conflictiva del factor tiempo por la irrupción desde el último cuarto del siglo pasado en los centros de Educación Primaria ${ }^{3}$ del modelo de jornada escolar de sesión única (en adelante JU), que concentra toda la actividad lectiva en periodo matinal, frente a la modalidad de sesión partida que distribuye el horario lectivo en sesiones de mañana y tarde (en adelante JP) ${ }^{4}$. El conflicto de las jornadas, persistente desde entonces, ha modificado la consideración de la variable tiempo que ha dejado de interpretarse como un elemento neutro en la organización escolar, para pasar a constituirse en el "supuesto" elemento clave de la calidad de la educación, si nos atenemos a las argumentaciones expuestas en los conflictos de las jornadas lectivas (FERNÁNDEZ ENGUITA, 2001; CARIDE, 1993, 2005).

Pero la preocupación por los tiempos escolares se deja sentir progresivamente en el marco de las políticas educativas de diversos países del mundo, mostrándose el interés por revisar las diferentes unidades cronológicas en que se enmarca y que imprime la institución escolar (calendario académico, jornada lectiva, sesión de aula, periodos vacacionales, ritmos escolares...). Buena

3 La Educación Primaria se corresponde con los 6 primeros años de los 10 en que se organiza la escolaridad obligatoria en España. Se comienza el primer curso a los 6 años de edad y se finaliza a los 12 , continuando a partir de aquí los siguientes 4 años en el nivel de Educación Secundaria Obligatoria (ESO).

4 La jornada lectiva diaria en Educación Primaria en España es de 5 horas. En la modalidad de sesión partida la distribución más extendida es de 3 horas por la mañana y 2 por la tarde. En sesión única las 5 horas se desarrollan en sesión matinal. 
muestra son las iniciativas emprendidas en Brasil alrededor de la educación integral que se muestran en este número o el reciente debate auspiciado por el Ministerio de Educación en Francia con la puesta en marcha a lo largo del año 2010 (entre junio y setiembre) de una Consulta Nacional sobre los Ritmos Escolares con numerosos e interesantes documentos sobre el debate.

\section{Tiempos, escuela y socialización}

El tiempo escolar es cuantitativa y cualitativamente uno de los tiempos sociales más relevantes, hasta el extremo de condicionar y determinar la organización de todas las demás temporalidades. Las niñas y niños suman diariamente un importantísimo número de horas consagradas esencialmente a la escuela, tanto en el tiempo propiamente escolar - horario obligatorio- como en el que Clarisse (2001) denomina periescolar -tareas para casa, clases particulares, desplazamientos-. Si se realiza una proyección de la inversión horaria cotidiana en semanas, días, meses y años, facilmente apreciaremos que una tercera parte de la vida transcurre bajo los influjos temporales de la institución escolar.

La dominancia cualitativa del cronos escolar va transformando bajo su disciplina, rigor y sanción todos los tiempos y espacios de las niñas y niños, de modo que tanto los que progresan sin dificultad, como los que tienen problemas académicos, ven su vida cotidiana totalmente impregnada de disciplina y responsabilidad escolar: clases de refuerzo, supeditación de cualquier actividad de ocio a los resultados y tareas académicas, etc. En palabras de Zaffran (2001, p. 19) se produce una "complejización y difusión del orden escolar y de los modelos que vehicula más allá de la escuela misma".

En definitiva, el tiempo escolar funciona como un regulador (material y funcional, además de simbólico) que incide en las posibilidades y límites de la socialización infantil, al marcar o definir buena parte del discurrir de las niñas y niños por los demás tiempos y espacios sociales. Compara el autor este proceso a un transitar interactivo entre las distintas islas de un archipiélago, de las que la más extensa y central es la escuela, por la importancia de los juegos sociales que representa. La socialización sólo es posible a través de los sucesivos desplazamientos entre cada una de las esferas -las islas-, correspondiéndole al tiempo escolar la articulación del paso entre las mismas: de la escuela a la familia; de esta a los amigos; de los amigos a la comunidad; de aquí nuevamente a la escuela...y así sucesivamente. En este discurrir espacio-temporal se van construyendo y desempeñando los diversos roles que nos conforman como personas y ciudadanos. 
Sin embargo, a pesar de la relevancia que el tiempo escolar tiene en la vida de las personas apenas se acompaña de una reflexión profunda en sus características y singularidades, manteniendo patrones totalmente obsoletos y disfuncionales. Porque el de la escuela es, en líneas generales, un tiempo rígido, lineal, monocorde, obsoleto, estereotipado y fuertemente resistente, cuando no impasible, a los cambios y necesidades sociales (CARIDE, 1993, 2005; FERNÁNDEZ ENGUITA, 2001; ZAFFRAN, 2001; CLARISSE, 2002; MORÁN; CARIDE, 2005). En definitiva, una larga lista de características opuestas a las que enmarcan el desarrollo de un proceso educativo de calidad que precisa de un concepto flexible del tiempo -y también del espacio-; de un tiempo policrónico rico, flexible, abierto y permeable que no cercene las posibilidades de aprender entre las agujas impenitentes de un cronómetro.

Y obviando o dejando a la margen este conjunto de temas inquietantes, en España la escuela centró su atención a lo largo de los últimos veinticinco años en la modificación de la jornada escolar, convertida en punto neurálgico de la educación Infantil y Primaria. Alrededor de tres décadas de tensos debates en el seno de las comunidades educativas, bajo la observación de la Administración que en la mayor parte de las Comunidades Autónomas se situó a la margen del conflicto, sin querer o atreverse a mediar y arbitrar decididamente en esta cuestión. La organización o reorganización de la jornada escolar, la adopción de una modalidad de sesión única, se abordó, y creemos que continúa abordándose, con grandes dosis de visceralidad, corporativismo interesado y con escasa perspectiva global de análisis: ni de los aspectos que subyacen en la misma, de las consecuencias derivadas de determinadas decisiones, o lo que es más grave, de la consideración de la infancia, sus intereses y necesidades.

El alboroto que se continúa produciendo alrededor de esta cuestión es síntoma claro de su alcance social, y de la dificultad que entrañan los acuerdos entre las partes implicadas. En definitiva, una clara muestra del importante significado de los tiempos educativos en el conjunto de los tiempos sociales, y concretamente, la problemática de los horarios y su conciliación en la vida familiar, laboral, institucional, etc.

\section{El proceso investigador}

Los antecedentes mencionados unidos a la trayectoria del grupo SEPA en investigación sobre tiempos sociales, educativos y escolares, impulsaron la realización de una investigación (Tesis doctoral) con la finalidad de estudiar la 
incidencia del factor tiempo en el desarrollo de la vida cotidiana de las niñas y niños de Educación Primaria en sus contextos familiar, extrafamiliar y escolar, tomando como principal referencia para el análisis la modalidad de jornada lectiva del centro educativo al que acuden.

Las aportaciones de los estudios precedentes (CARIDE, 1993, 2005) nos condujeron a formular como hipótesis de trabajo los siguientes postulados:

- Los procesos de socialización infantil están mediatizados por la escuela y sus ritmos de actividad, existiendo diferencias significativas en función de la modalidad de jornada escolar (sesión partida/sesión única) en el marco de la vida cotidiana. Estas diferencias afectan a los ámbitos familiar (posibilidades de convivencia, interacción entre iguales, así como al desarrollo de prácticas y hábitos de salud infantil) y extra-familiar (organización y uso del tiempo libre; otras oportunidades educativas fuera de la escuela).

- Las modalidades de jornada lectiva suscitan diferencias en la vida escolar, con desigualdades entre ambas en el tiempo disponible para el trabajo escolar, y en su organización y desarrollo.

- Las modalidades de jornada lectiva no se acomodan de modo semejante a los diversos contextos socioeconómicos y culturales de las familias ni de los centros educativos.

En el proceder metodológico, de carácter cuantitativo con finalidad descriptiva, comparativa, analítica e interpretativa, se empleó como instrumento de recogida de información un cuestionario mixto de 91 ítems ordenados en 13 bloques temáticos más un apartado final de preguntas abiertas. Presenta un recorrido a través de las distintas secuencias que componen un día en la vida de una niña o niño. Siguiendo las agujas del reloj, se inicia con la descripción de la primera hora de la mañana (hora de levantarse, de desayunar, desplazamiento hasta la escuela...), para ir deslizándose por las diversas rutinas, acontecimientos, y circunstancias cotidianas (la escuela, los recreos, las horas de comida, las actividades extraescolares, los deberes...) hasta la hora de acostarse (la cena, la compañía familiar, la televisión...).

Para la aplicación del cuestionario se diseñó un muestreo básico de conglomerados por cuotas -monoetápica en el caso de los centros y polietápica en el del alumnado- que concluyó en una muestra de 884 alumnas y alumnos de Educación Primaria pertenecientes a 21 centros educativos. Las características del cuestionario, que requerían cierto nivel de comprensión y valoración, aconsejaron su aplicación en alumnado de $4^{\circ}$ curso (9-10 años) y $6^{\circ}$ curso (11-12 años). La configuración atendió a las cuotas establecidas en función de la modalidad de jornada lectiva, contexto o zona de emplazamiento y titularidad del centro. El cuadro $\mathrm{n}^{\circ} 1$ recoge las características generales del proceso muestral.

En el análisis de los datos, en función de su presentación en frecuencias, se 
empleó la prueba no paramétrica de inferencia estadística $\chi^{2}$ para analizar diferencias entre las categorías establecidas según los objetivos de la investigación.

- Universo: 140.589 alumnas/os de Educación Primaria en Galicia

- Tipo de muestreo: conglomerados por cuotas

Monoetápica: centro

- Modalidad de jornada (partida/única)

- Hábitat de emplazamiento (rural/semiurbano/urbano)

- Titularidad (pública/privada)

Polietápica: alumnado

$-4^{\circ}$ curso

- $6^{\circ}$ curso

- Tamaño de la muestra: 884 alumnas/os 21 centros escolares

- 409 jornada partida

- 475 jornada única

- Tratamento estadístico: $\chi^{2}$

- Cronología del trabajo de campo: marzo-mayo de 2002

CUADRO 1 - Características de la muestra de investigación

\section{Organización y empleo del tiempo en la vida cotidiana. Resultados obtenidos}

Nos referimos con esta denominación al conjunto de acontecimientos entre los que transcurre un día de las y los pequeños, excluidos los desarrollados en la escuela. Concretamente se contemplan: el conjunto de actividades vinculadas a las posibilidades de convivencia e interacción familiar; el desarrollo de hábitos saludables de alimentación y reposo; el empleo del tiempo libre de disposición en actividades extraescolares, juego y acceso a la televisión.

\section{Tiempo de convivencia familiar}

De la institución familiar, inmersa en un progresivo proceso de remodelación de tipologías y modificación de funciones, se cuestiona reiteradamente su 
efectividad en el desarrollo del proceso de socialización primaria. Tal es así que ha sido definida como una "unidad mínima de consumo orientada a la crianza -y cada vez menos a la socialización- de los hijos" (FERNÁNDEZ PALOMARES, 2003, p. 217). No obstante, a pesar de la delegación de una buena parte de sus responsabilidades en otras instituciones, su estructura y características, así como las intensidad y calidad de las interacciones establecidas entre sus miembros, determinan en gran manera los procesos de socialización infantil. Y a su vez, como hemos señalado, las posibilidades de interacción, se encuentran moduladas por el influjo de la escuela y su organización temporal. Uno de los objetivos prioritarios de muchas familias es conseguir compaginar los diferentes ritmos familiares (horarios laborales de padre y/o madre, horarios escolares, horarios de actividades compartidas por los integrantes del núcleo familiar así como de actividades personales de sus miembros...) para posibilitar mayores y mejores ocasiones para la convivencia. Esta ha sido una de las ventajas que se han querido vincular a la jornada de sesión única, al disponer los pequeños de toda la tarde -teóricamente- libre; pero para ser veraz esta afirmación requiere que los progenitores dispongan igualmente de este tiempo liberado para compartirlo, lo que ineludiblemente está vinculado a disfrutar de determinadas condiciones socioeconómicas que no son generalizables al conjunto de familias. Veamos los resultados que obtenemos al respecto:

- Ocupación laboral de padres y madres: En la investigación constatamos que las familias de alumnado de jornada única (JU) desarrollan en mayor medida que los de Partida (JP) puestos de trabajo vinculados a un desarrollo socioeconómico y cultural medio y medio/alto apreciándose diferencias significativas al comparar las profesiones de los padres y de las madres en ambos tipos de jornada. Las tres categorias más señaladas son: profesiones liberales/ técnicos (27,9\% en JU; $13,5 \%$ en J.P.); sector secundario (22,1\% en JU; $36,7 \%$ en J.P.); sector servicios (13,0\% en JU; $15 \%$ en JP). Este resultado corrobora el obtenido en investigaciones precedentes (FERNÁNDEZ ENGUITA, 2001) que lleva a definir la implantación de JU como una demanda de clases media/ media-alta.

- Horario laboral de los padres: La jornada laboral predominante entre padres y madres es con horario partido, lo que implica que deben acudir a su trabajo por la mañana y continuar por la tarde $(66,9 \%$ de los padres; $44,0 \%$ de las madres). Por lo tanto la posibilidad de concordancia entre jornada laboral y escolar señalada como argumento a favor de la sesión única se reduciría, además de quienes no trabajen fuera del hogar, a un $13,4 \%$ de los padres y el $33,4 \%$ de las madres de alumnado en JU cuyo horario laboral es exclusivamente por la mañana.

La enfermedad del tiempo, que asola destacadamente a las sociedades económicamente más desarrolladas, está haciendo crecer a muchas niñas y 
niños en condiciones de "ejemplares aislados" hijos de padres y/o madres esporádicos que aportan pequeñas dosis diarias de afecto y atención, y que modelan su conducta con experiencias facilitadas primordialmente por los medios audiovisuales y redes virtuales que sí están permanentemente a su disposición. Los resultados obtenidos corroboran esta situación deficitaria a pesar de los innumerables esfuerzos familiares por conseguir la combinación adecuada entre las variables de vida de los miembros de la familia para lograr incrementar ese tiempo imprescindible de vivir compartidamente.

- Tiempo de encuentro familiar. El final del día (68,8\% señala "la noche") se confirma como el momento de coincidencia familiar para el alumnado de ambas modalidades de jornada, sin que se aprecien diferencias en las oportunidades de convergencia en el domicilio en otros horarios como mediodía o tarde que se señalan como teóricamente favorecidos para el reencuentro con la modalidad de jornada única. Incluso a mediodía, a la hora de comer, se reúnen el grupo familiar del $45,1 \%$ de la muestra, sin diferencias significativas en función del tipo de jornada (46,1\% JU; 44,0\% JP).

\section{Hábitos de alimentación y descanso}

Las prácticas de alimentación de la infancia escolarizada en España son, en términos globales, deficientes tanto por el cambio producido en los patrones tradicionales dietéticos desplazados por la inmediatez y comodidad de la comida rápida hipercalórica, como por el sedentarismo o inactividad física que promueve el consumo desorbitado de pantallas (televisión, ordenadores...), a lo que hay que añadir el retroceso, disminución o practicamente ausencia de pautas de socialización familiar en lo relativo a normas y hábitos de comportamiento y supervisión alimentaria (TOJO; LEIS, 1999). Sea como fuere, en muchos hogares los momentos de comida se han ido transformando de oportunidades de reunión, encuentro e intercambio familiar a fugaces momentos de ingesta de alimentos sin casi más requisito que terminar pronto y no siempre con la presencia de adultos.

- Tiempo para la alimentación. Los resultados obtenidos ratifican una situación deficitaria en cuanto a los tiempos dedicados y número de miembros de la familia que comparten los momentos dedicados a la alimentación. El desayuno y la comida transcurren en intervalos de tiempo especialmente cortos y, por lo tanto, difícilmente satisfactores de las condiciones necesarias para una dieta sana y equilibrada, ni por la cantidad posible de alimentos a ingerir, ni por la velocidad con que 
se efectúan. La mayoría del alumnado de la muestra $(72,6 \%)$ toma su desayuno en un tiempo de entre 5 y 10 minutos. En el Estudio Enkid, (SUÁREZ et al., 2000), se subraya la correlación entre desayunos de corta duración y bajas ingestas; señalan cómo en un tiempo inferior a 10 minutos sólo se cubren el 13,8\% de las ingestas dietéticas de referencia diaria, lo que sucedería con las tres cuartas partes del alumnado de Educación Primaria encuestado. La comida de mediodía, que se realiza mayoritariamente en el hogar, transcurre para un $42 \%$ en un tiempo máximo de 15 minutos, señalado en mayor porcentaje por el alumnado de JP (50,5\%) que tras comer debe regresar al colegio para continuar la jornada lectiva, que de JU (35\%). Los datos obtenidos apuntan a la mejora de estas condiciones cuando la comida se realiza en los comedores escolares, donde las mayores frecuencias se recogen en el intervalo 30-60 minutos (57,9\%). Las deficiencias señaladas en el hogar son subsanadas en los comedores escolares donde no sólo se siguen dietas y pautas nutritivas elaboradas por especialistas, sino que se acompaña educativamente y se supervisa siempre por adultos el momento de comer.

Las carencias en el descanso nocturno, cada vez más extendidas entre la infancia, son causa de alteraciones psíquicas y somáticas, con consecuencias, entre otros aspectos, en el rendimiento escolar. Diversos autores han analizado estos factores (FOTINOS; TESTU, 1996; RANDAZZO, 1998; CHALLAMEL, 2001; CARNO et al., 2003; CLARISSE et al., 2004; MEIJER; VAN DEN WITENBOER, 2004), coincidiendo en apuntar a la organización familiar como determinante en la consolidación de hábitos saludables de descanso, pero sin olvidar la supeditación de la misma a los "sincronizadores sociales", entre los que se sitúa en lugar destacado el tiempo escolar y sus demandas. El horario de entrada al colegio, la sincronía entre este horario y el del comienzo de la jornada laboral paterna y/o materna, el tiempo invertido en el trayecto al centro en función de la distancia con el domicilio... determinan el horario para levantarse de la cama y - para salvaguardar las necesidades de reposo de la infancia- el de acostarse y dormir.

- Tiempos para el descanso. Siguiendo la tónica negativa de los tiempos para la alimentación, los resultados obtenidos respecto a los hábitos de descanso muestran como un importante porcentaje de las y los pequeños de la muestra $(38,9 \%)$ no duermen el periodo de sueño aconsejables a estas edades -ente 10 y 11 horas- (NATIONAL SLEEP FOUNDATION), con la consiguiente acumulación de deudas en la cuenta de sueño que, pese a la falsa creencia popular, no se recupera durmiendo más durante los fines de semana y vacaciones. Las deficiencias en el descanso nocturno se identifican en un 43,9\% del alumnado de JP y en un 50,4\% del alumnado de JU (cuyo horario de entrada al colegio es, en términos generales, 1 hora antes que los de jornada partida). Por otra parte habría que añadir otros aspectos cualitativos del reposo nocturno, como son la 
sensación de cansancio y facilidad para el sueño. Destacamos en este sentido que un $50,5 \%$ de la muestra indica tardar mucho $(21,9 \%)$ o bastante $(28,6 \%)$ en quedar dormidos, lo que disminuiría el ya escaso tiempo calculado.

\section{Los tiempos de libre disposición}

El tiempo libre de las niñas y niños, lejos de favorecer los objetivos de descanso, diversión y desarrollo personal que deberían corresponderle, se transforma progresivamente en un tiempo esclavo que acaba por incrementar las presiones sobre los horarios de los pequeños, cada vez más recargados. Un tiempo imprescindible sobre todo si tenemos en cuenta que los niños y adolescentes españoles "son, ante todo, unos intensos consumidores de escuela... viven recluidos en estos centros durante quince años, constreñidos al abandono de la libertad que implica la condición de alumno [...] la reclusión escolar hace que, a menudo, se olvide que el ocio es un necesario contrapunto de la vida escolarizada" (CUENCA, 2000, p. 302). Pero su libertad se fue limitando, como recuerda Tonnuci (1997), por tres motivos: para hacer frente a los peligros de la calle, lo que conduce al "encierro infantil"; para atender la necesidad de custodia en ausencia de los padres y madres, lo que convierte a estas actividades en obligación más que opción personal; finalmente, por la mejora de las condiciones económicas, promotora de mayor inversión en el capital cultural de los hijos, y por lo tanto de "aprovechamiento" del tiempo de disposición personal.

En este tiempo libre debería ocupar un lugar prioritario la que sin duda es -cómo decía el filósofo francés Montaigne- la actividad más seria de los niños y fuente insustituible de aprendizajes: el juego. Por el contrario, asistimos a una restricción espacio-temporal de sus posibilidades, tanto por la limitación de ocasiones que conlleva un tiempo libre excesivamente planificado y rentabilizado, como por la ausencia o limitación de espacios para jugar.

Sí tienen cabida y relevancia en el tiempo de libre disposición de la infancia las actividades extraescolares, entre otras causas por haberse convertido en requisito obligatorio para la concesión de la modalidad de jornada lectiva de sesión única; así inicialmente se presentan magnificadas en los proyectos que los centros presentan y con promesas de expansión que, por diversos motivos, no llegan a concretarse en la realidad. Estas actividades han venido progresivamente mercantilizándose y alejándose de los centros escolares, de la mano de un florecido sector de empresas especializadas, marco idóneo para la clase media, pero vedado, inapropiado, sin interés e incluso sin posibilidades de aprovecha- 
miento para los grupos sociales de menores recursos económicos y culturales.

- Tiempo de actividades extraescolares. Los datos obtenidos permiten constatar una alta participación $(74,8 \%)$ en actividades extraescolares con diferencias significativas favorables a la JU, tanto en los porcentajes de asistencia (JU 80,8\%; JP 67,7\%) como en el promedio de días y horas semanales dedicadas a las mismas (JU 3 días/ 6 horas; JP 2 días/2 horas). La actividad extraescolar se focaliza en el deporte $(87,4 \%)$ a enorme distancia de otras opciones: actividades artísticas $(35,0 \%)$, culturales $(32,6 \%)$, musicales $(31,5 \%)$, informática $(30,1 \%)$. Otro aspecto relevante es que en ambas modalidades acuden fundamentalmente a la oferta exterior a la escuela. Cabe destacar que entre el alumnado que no realiza actividades extraescolares (14,7\% JU y un $24,2 \% \mathrm{JP})$ se anotan como razones en primer lugar la carencia de tiempo y en segundo lugar la "no gratuidad" de la oferta; esta cuestión señala directamente a limitaciones económicas que pueden ser fuente de discriminación y desigualdad si no se ponen en marcha mecanismos correctores.

- Tiempo de juego. El juego, que debería ser la actividad primordial si este tiempo de disposición personal realmente respondiese al adjetivo "libre", está espacialmente restringido. Los resultados obtenidos indican la tendencia a jugar fundamentalmente en espacios cerrados (en la propia casa $60,8 \%$; en casa de amigas/os 39,7\%) frente a los abiertos (en la calle 33,7\%; en el parque $30,6 \%)$. Los datos corroboran la tesis de la "casa fortaleza" frente los peligros externos que describe Tonnuci (1997), y que incide negativamente en las posibilidades de movilidad autónoma de la infancia y de socialicación con el grupo de iguales en momentos de ocio. La disponibilidad temporal se concreta para la mayoría en dos horas por día sin diferencias entre modalidades de jornada. Sin embargo los de JP valoran significativamente peor que los de JU el tiempo del que disponen para jugar, lo que es consecuente con su permanencia en el centro escolar en horario de tarde.

- Tiempo de televisión. Ver televisión es la (in)actividad primordial en el tiempo de libre disposición, y de la que sin duda el principal peligro, como indican Anderson y Wilkins (2000) no está tanto en la conducta que provoca como en la que inhibe. Es una rutina ineludible en la cotidianeidad de las pequeñas y pequeños sin que la modalidad de jornada escolar determine diferencias significativas en la frecuencia con que se ve, en la banda horaria habitual, en el tiempo dedicado durante la semana y fin de semana o en el tipo de programas contemplados. La mayoría de la muestra ve televisión diariamente (66,9\%), y todas las bandas horarias parecen propicias -y lo que es peor, posibles- tanto en los días de escuela, como en el fin de semana. Se constata la tendencia señalada en otros estudios (GARCÍA 2000, URRA et al., 2000) que revelan mayor dedicación cuanto más se incrementa el tiempo libre: los sábados y domingos, un $37,2 \%$ de la muestra permanece ante el televisor más de tres horas diarias. 
También se ratifica la ausencia de educación y control familiar en el consumo televisivo, expresado en la posibilidad de ver todo tipo de programas. Entre los preferidos figuran especialmente dibujos animados, series y películas, muchas de las cuales comienzan su emisión pasadas las 22:00 horas y con una duración por término medio de 90 minutos.

\section{Organización y uso del tiempo en la vida escolar y peri-escolar. Resultados obtenidos}

El tiempo escolar se ha convertido en una variable hegemónica que absorbe una parte importantísima de las jornadas y esfuerzos de las niñas y niños y de sus familias, para poder responder a los requerimientos y servicios que genera. Pero la escuela no sólo exige, gestiona y distribuye horas, sino que también socializa a los pequeños en el aprendizaje de las temporalidades según sus propios criterios. Enseña "su" tiempo, su concepto y expresión de un tiempo monocrónico, inmóvil, fijo y rígido que, no sólo está lejos de acomodarse a las nuevas necesidades educativas y sociales, sino que además apenas respeta las evidencias y criterios científicamente avalados, fundamentalmente desde la Cronobiología y la Cronopsicología, para optimizar el proceso educativo atendiendo a las características de los pequeños humanos. En la organización escolar continúan prevaleciendo los intereses laborales y económicos antes que los de las alumnas y alumnos en la distribución de horarios, de asignaturas, de periodos de descanso, etc... que continúan siendo "consecuencia más bien de la aplicación de criterios socioeconómicos que de los derivados del conocimiento del hombre como ser vivo" como indicaba Asensio (1993, p. 107) casi veinte años atrás.

Abordamos en este apartado los resultados relativos a aspectos organizativo-institucionales de la escuela vinculados al factor tiempo, así como la percepción que el alumnado tiene del desarrollo de actividades académicas, relacionales y de participación en el transcurso de la jornada lectiva escolar.

- Horarios de entrada y salida: Entre los centros de cada modalidad de jornada se observa una gran variabilidad en los horarios de entrada y salida. Los que desarrollan la jornada lectiva de sesión única comienzan la actividad en el intervalo comprendido entre 8:30-9:30 y la terminan entre las 13:40-14:30; el horario más generalizado es iniciar a las 9:00 h. y terminar la actividad docente a las 14:00. Los centros de sesión partida, por la mañana comienzan en el intervalo 9:20-10:10 y terminan entre las $12: 45$ y las 13:35. Por la tarde el retorno a las aulas se produce entre las 14:45 y las 15:30 y el remate de actividad lectiva 
tiene lugar entre las 16:30 y las 17:30 observándose una tendencia a prolongar la sesión de mañana y acortar la de tarde.

- Uniformidad horaria de la jornada escolar. Internamente no se realiza una diversificación horaria que permita adaptarse mejor a las características y edades del alumnado, manteniendo horarios uniformes dentro de la jornada escolar tanto para todo el $2^{\circ}$ ciclo de Educación Infantil (con edades de 3 a 5 años), como para la Educación Primaria (con edades de 6 a 12 años). Es una de las muestras de la rigidez, inmovilidad y monocronía de la organización escolar, a la que nos hemos referido más arriba.

- Tiempo de recreo. Pausa para descansar del trabajo intelectual, jugar, ingerir alimentos, los recreos son momentos imprescindibles que deben estar perfectamente planificados y situados en el conjunto de la jornada escolar. Sin embargo, la uniformidad observada es extensible al tiempo de recreo que, independientemente de la modalidad de jornada (ya sean 5 horas o tres horas de clase por la mañana), se concretan en una única pausa de alrededor de media hora. En los centros de JU esta pausa se introduce después de haber realizado ya dos tercios del volumen horario de la jornada lectiva (alrededor de 2:45h.). En los de JP tras haber desarrollado 1:45 h. restando despues del recreo un tiempo semejante. Por otra parte no es infrecuente perder parte del tiempo de recreo por emplearlo en tareas académicas; esta circunstancia negativa es más frecuentemente señalada entre el alumnado de sesión única, reiterándose entre las causas la "falta de tiempo para finalizar trabajos escolares" (explicaciones, correcciones, deberes...).

- Empleo del tiempo escolar. Una cuestión relevante en el estudio del tiempo escolar es el empleo que se hace del mismo, lo que Cavet (2011) denomina la "caja negra" de la escuela o si se quiere "la escuela por dentro". En relación a esta cuestión hemos solicitado del alumnado su valoración sobre la disponibilidad temporal para realizar actividades que hemos agrupado en tres categorías: actividades específica y tradicionalmente académicas (estudiar, aclarar dudas, trabajar en grupo...); actividades relacionales (interacción con los diferentes miembros de la escuela, resolver problemas personales); actividades de participación en la vida del centro (organización y participación en fiestas y juegos; decorar/cuidar la escuela; descansar...) indispensables en un concepto integral de educación al hacer posible el ejercicio y expresión de aprendizajes que nos construyen como persona, como ciudadano, y como miembro de una comunidad.

Las actividades académicas alcanzan una valoración positiva (disponer de bastante o mucho tiempo) en cuanto a las acciones que implican un manejo y organización personal y negativa en los que supone realizar trabajos compartidos y utilizando diferentes espacios de la escuela (p.e. ir a la biblioteca). Es decir, no tienen opción temporal las propuestas vinculadas a un concepto de escuela diferente, renovada, y de horizontes más amplios que los muros físicos y psico- 
lógicos del espacio-aula. Las actividades relacionales obtienen una valoración positiva del tiempo a disposición para la interacción con los compañeros de aula y el profesor/a-tutora, pero negativa para posibilitar la relación con otro aluumnado y profesorado del centro. Las actividades de participación en la vida del centro prácticamente parecen no existir en el desarrollo cotidiano escolar.

Esta perspectiva tan escasa en cuanto a otras posibilidades de uso del tiempo en la escuela más allá de las actividades clásicas y el espacio-aula, ha sido corroborada en investigaciones posteriores realizadas por el grupo SEPA ${ }^{5}$.

- Tareas escolares para realizar en casa y asistencia a clases particulares. Los deberes escolares y la asistencia a clases particulares son actividades ubicadas en un tiempo intermedio entre el de libre disposición y el escolar que Clarisse (2002) denomina peri-escolar, caracterizado por estar bajo el influjo y las obligaciones derivadas de la escuela. Concretamente el 56,8\% del alumnado de la muestra tiene que realizar diariamente en casa tareas escolares que le son encomendadas (más frecuentemente en el alumnado de JP), a las que destina, por término medio, entre 30 y 60 minutos (mayor inversión temporal en el alumnado de JU). Uno de cada cinco alumos asiste, además, a clases particulares con una duración de 1 hora como promedio; el alumnado de JP acude en mayor porcentaje pero con menor frecuencia semanal (2 días); los de JU completan practicamente a dirario la jornada escolar con esta actividad lectiva paralela.

\section{Para un tiempo de (y con) futuro}

Finalmente, situando el tiempo de la infancia en primer plano y en el intento de superar la focalización sesgada de los tiempos educativos y escolares a una dicotomía de modalidades de jornada, queremos anotar algunas reflexiones en clave de presente y futuro, urgiendo a un nuevo enfoque en el conflicto de las temporalidades de las niñas y niños.

La primera cuestión será abrir el angular para la observación, porque la cuestión que se dirime no es un problema de horario escolar, sino de los tiempos sociales y educativos en su conjunto, por tanto una observación desde la globalidad de los tiempos de las niñas y niños y sus familias, así como de la necesidad de su ordenación, conjunción y coordinación.

La segunda cuestión incorpora la complejidad, pero también la riqueza que precisa el análisis de los problemas sociales: se requiere una observación multidisciplinar, integrando el conjunto de perspectivas (sociológica, médica,

5 Tiempos escolares y tiempos de ocio: escolarización y vida cotidiana de la infancia en la sociedad red. Director: J.A. Caride. Plan Nacional I+D+i 2005-2008. 
pedagógica, psicológica, política...) que permitan organizar y desarrollar propuestas de acción con el máximo respeto a las características, posibilidades, necesidades y derechos del alumnado. Las niñas y niños como punto de partida en el análisis y como punto central de retorno en la toma de decisiones.

La tercera, consecuencia de las anteriores, es que no son "partes" de la escuela las que deben ser revisadas, sino la escuela misma en su conjunto: sus proyectos educativos y acciones, su sentido y funciones, a fin de salvar, o cuando menos reducir, su escasa respuesta a las demandas sociales que viene reiterando la rigidez y el anacronismo de la organización escolar. Una escuela, señalan (VASQUES; MARTÍNEZ, 1996), de rituales fundamentados más que en exigencias naturales y científicas, en decisiones políticas e históricas, que se consideran "dadas" en la institución escolar y son asumidos sin apenas cuestionamiento

La cuarta, avanzar en la consolidación de redes locales socioeducativas con planes, programas y proyectos liderados por la administración local, en los que todas las instituciones e iniciativas comunitarias actuen coordinada y cooperativamente en la mejora de la calidad de la educación de la ciudadanía, y muy especialmente de las y los menores. Propuestas que atiendan a los cambios sociales y a las nuevas necesidades que de ellos se derivan en la atención a la infancia; que dispongan medidas preventivas de desigualdades y discriminaciones en el ejercicio de los derechos y en el crecimiento como persona y ciudadano; que avancen la conciliación de intereses laborales, familiares y escolares.

Son todos ellos aspectos sustanciales para procurar una inserción armoniosa de los tiempos de la escuela en la vida cotidiana de la infancia, y no al revés, supeditando a los pequeños en la cuadrícula insensible de una organización cartesiana. Propuestas que sin duda permitirían avanzar en la mejor disposición y uso de un tiempo para los afectos, un tiempo como libertad, y un tiempo como derecho para la construcción personal sin restricción horaria.

\section{REFERENCIAS}

ANDERSON, J. E WILKINS, R. ¡Que no te atrape la pantalla! Consejos para no abusar de la TV y del ordenador. Madrid: Santillana, 2000.

ASENSIO, J. P. Cronobiología y educación. In: FERMOSO, P. (Ed.). El tiempo educativo y escolar. Estudio interdisciplinar. Barcelona: PPU, p. 75-109, 1993.

BAUMAN, Z. Tiempos líquidos. Vivir en una época de incertidumbre. Madrid: Tusquets Eds., 2007. 
CAVET, A. Rythmes scolaires: pour une dynamique nouvelle des temps éducatifs. Dossier d'actualité, n. 60, février 2011. Disponible en: <http://ife.ens-lyon.fr/vst/DA/ ListeDossiers.php?/60-fevrier-2011.php>. Acceso en: 15/09/2011.

CARDÚS, S. Propostes d'intervenció per a la conciliaciód'horaris familiars, escolars i laborals: informe final. Barcelona: Generalitat de Catalunya-Departament de Benestar i Familia, 2003.

CARIDE, J. A. (Dir.). A xornada escolar de sesión única en Galicia. Estudio avaliativo: conclusións xerais e criterios de actuación. Santiago de Compostela: Xunta de Galicia-Consellería de Educación e Ordenación Universitaria/Instituto de Ciencias da Educación da Universidade de Santiago de Compostela, 1993.

. (Dir.). A xornada escolar de sesión única en Galicia: avaliación da súa implantación edesenvolvemento nos centros de Educación Infantil, Primaria e Públicos Integrados. Santiago de Compostela: Xunta de Galicia, 2005.

CARNO, M. A. et al. Developmental stages of. Sleep from birth to adolescence, common childhood sleep disorders: overview and nursing implications. Journal of Pediatric Nursing, v. 18, n. 4, p. 274-283, 2003.

CLARISSE, R. De l'évaluation des rythmes de l'enfant en milieu scolaire à lévaluation des aménagements du temps scolaire. Quelles perspectives pour les chronopsychologues?. [Ponencia] Madrid: Seminario Internacional Universidad Complutense Ritmos Psicológicos y Jornada Escolar. 2002. Disponible en: <http://forteza.sis.ucm.es/profes/ juanfran/crono/sic >. Acceso en: 12/05/2004.

. Étude comparative des durées et des horaires du sommeil nocturne d'enfants de cinq à dix ans selon leur âge et leur environnement socio-économique. Archives de pédiatrie, 11, p. 85-92, 2004.

CHALLAMEL, M. J. Sleep in school-age children. Acta paediatrica, v. 90, n. 12, p. 1365-1367, 2001.

CUENCA, M. El fenómeno social del ocio. In: FERMOSO, P.; PONT, J. (Eds.). Sociología de la educación. Valencia: Nau Llibres, 2000, p. 291-311.

FERNÁNDEZ ENGUITA, M. La jornada escolar. Análisis y valoración de los procesos, los efectos y las opciones de la implantación de la jornada continua. Barcelona: Ariel, 2001.

FERNÁNDEZ PALOMARES, F. Sociología de la educación. Madrid: Pearson Educación, 2003.

FOTINOS, G.; TESTU, F. Aménager le temps scolaire. París: Hachette Éducation, 1996.

GARCÍA, M. C. Televisión, violencia e infancia. El impacto de los medios. Barcelona: Gedisa, 2000.

MARRAMAO, G. Kairós. Apología del tiempo oportuno. Barcelona: Gedisa, 2008. 
MEIJER, A. M.; WITTENBOER, G. L. Van Den. The joint contribution of sleep, intelligence and motivation to school performance. Personality and Individual Differences, v. 37, p. 95-106, 2004.

MORÁN, M. C. A xornada escolar na vida cotiá da infancia. Análise da incidencia dasmodalidades de sesión 'partida-única'nos procesos de socialización infantil en Galicia. Tese Doutoral. Universidade de Santiago de Compostela/Servizo de Publicacións e Intercambio Científico, 2005.

MORÁN, M. C.; CARIDE J. A. La jornada escolar en la vida cotidiana de la infancia. Cuadernos de Pedagogía, v. 349, p. 64-69, 2005.

NATIONAL SLEEP FOUNDATION. How Much Sleep Do We Really Need? Disponible en: < http://www.sleepfoundation.org/article/how-sleep-works/how-much-sleep-do-we-really-need $>$. Acceso en: 20/12/2011.

RANDAZZO, A. C. et al. Cognitive function following acute sleep restriction in children ages 10-14. Sleep, 21, p. 861-868, 1998.

SUÁREZ, J. C. et al. Desayuno y rendimiento. In: SERRA, L.; ARANCETA, J. Desayuno y equilibio alimentario. Estudio enKid. Barcelona: Masson, 2000, p. 19-30.

TONUCCI, F. La ciudad de los niños. Un modo nuevo de pensar la ciudad. Madrid: Fundación Germán Sánchez Ruipérez, 1997.

URRA, J. et al. Televisión: Impacto en la infancia. Madrid: Siglo XXI, 2000.

VASQUES, A; MARTÍNEZ, I. La socialización en la escuela. Una perspectiva etnográfica. Barcelona: Paidós, 1996.

ZAFFRAN, J. Le temps scolaire, le temps libre e le temps des loisirs: comment réussir au collège français en s'engageant dans la course des temps sociaiux, Loisir et Société, v. 24, n. 1, 2001. Disponible en: <http://www.erudit.org/revue/ls/2001/v24/n1/000166ar.html>.

Texto recebido em 30 de março de 2012.

Texto aprovado em 02 de maio de 2012. 